\title{
Preparation of a Novel N,O-carboxymethyl Chitosan/Glycine- Modified Electrode and Electrochemical Determination of Uric Acid and Ascorbic Acid
}

\author{
Jun Li Zhang, Rongqiang $\mathrm{Li}^{*}$, Yan Zhang \\ College of Chemical and Pharmaceutical Engineering, Huanghuai University, Zhumadian 463000, \\ China \\ *E-mail: $\underline{\text { rqli@iccas.ac.cn, zjlwzq@yeah.net }}$
}

doi: $10.20964 / 2017.07 .33$

Received: 7 December 2016 / Accepted: 23 April 2017 / Published: 12 June 2017

\begin{abstract}
A N,O-carboxymethyl chitosan/glycine composite (NOCC-Gly-GC) membrane-modified electrode was prepared by coating a mixture of N,O-carboxymethyl chitosan (NOCC) and glycine (Gly) onto the surface of a glassy carbon electrode. Moreover, cyclic voltammetry was employed to characterize the electrochemical behavior of uric acid (UA) and ascorbic acid (AA) on the modified electrode. The NOCC-Gly-GC electrode was catalytically oxidized significantly, and the effective electrode area was improved with respect to that of the glassy carbon electrode. However, Gly and carboxymethyl chitosan not only increased the electrode area and active sites but also accelerated the electron transfer. In phosphate buffer solution ( $\mathrm{pH}$ 5.59), both AA and UA were sensitive to irreversible oxidation peaks with NOCC-Gly-GC electrode. A good linear relationship between oxidation peak current and concentration was observed. After the parallel determination of mixed liquid seven times, the relative deviation ratios of AA and UA were detected to be $2.1 \%$ and $3.6 \%$, respectively. These ratios indicated that the NOCC-Gly-GC electrode possessed electrochemical stability and reproducibility. The modified electrode exhibited well-separated oxidation peaks for UA and AA, with a potential difference of $341 \mathrm{mV}$. Thus, both simultaneous and individual measurements for UA and AA can be established. We expect the method to hold wide applications in real sample determination.
\end{abstract}

Keywords: N,O-carboxymethyl chitosan; glycine; NOCC-Gly-GC electrode; uric acid; ascorbic acid

\section{$\underline{\text { FULL TEXT }}$}

(C) 2017 The Authors. Published by ESG (www.electrochemsci.org). This article is an open access article distributed under the terms and conditions of the Creative Commons Attribution license (http://creativecommons.org/licenses/by/4.0/). 